\title{
Roles of Novel Reactive Cationic Copolymers of 3-Chloro-2-hydroxypropylmethyldiallylammonium Chloride and Dimethyldiallylammonium Chloride in Fixing Anionic Dyes on Cotton Fabric
}

\author{
Yikai Yu' ${ }^{1,2}$ and Yuejun Zhang ${ }^{1}$ \\ ${ }^{1}$ School of Chemical Engineering, Nanjing University of Science and Technology, Nanjing 210094, China \\ ${ }^{2}$ College of Chemistry and Chemical Engineering, Jiangxi Normal University, Nanchang 330022, China \\ Correspondence should be addressed to Yuejun Zhang, zhangyuejun68@163.com
}

Received 22 June 2012; Revised 1 November 2012; Accepted 9 November 2012

Academic Editor: Yufang Zhu

Copyright (C) 2013 Y. Yu and Y. Zhang. This is an open access article distributed under the Creative Commons Attribution License, which permits unrestricted use, distribution, and reproduction in any medium, provided the original work is properly cited.

The roles of novel reactive cationic copolymers (P(CMDA-DMDAAC)s) of 3-chloro-2-hydroxypropylmethyldiallylammonium chloride (CMDA) and dimethyldiallylammonium chloride (DMDAAC) in fixing anionic dyes on cotton fabric were studied by modern instrumental analysis technologies such as FT-IR spectra and SEM analysis, to achieve the new theoretical guides for the wide applications of those dye fixatives. The FT-IR spectra of the obtained insoluble-water color lakes verified that they could be formed from the electrostatic interactions of the $\mathrm{P}(\mathrm{CMDA}-\mathrm{DMDAAC})$ s with anionic dyes, which were further confirmed by the FT-IR analysis of the anionic dyes on dyeing cotton sample fixed by P(CMDA-DMDAAC)s. The FT-IR spectra of cotton samples fixed by $\mathrm{P}(\mathrm{CMDA}-\mathrm{DMDAAC})$ s showed the absorptions of $\mathrm{P}(\mathrm{CMDA}-\mathrm{DMDAAC})$ s and the signs similar to the formation of new ether linkage on cotton fabric even after being repeatedly washed, which were further confirmed by the SEM analysis of the fixed dyeing cotton samples. Thus, the reactive units (CMDA) of the obtained P(CMDA-DMDAAC)s could be expected to bring about the covalent bonds with the hydroxyl groups of cotton (cellulose) to form an ether linkage when fixing, resulting in the stronger interactions of $\mathrm{P}(\mathrm{CMDA}-\mathrm{DMDAAC})$ s with cotton fabric, as well as their electrostatic forces with anionic dyes to produce the insoluble-water color lakes, for the development of fastness of anionic dyes on cotton fabric.

\section{Introduction}

Cotton fabric is the most widely welcomed textile in the world, which is mainly made of cellulose [1]. Dyed cotton should have high colorfastness to usual repeated domestic launderings [2].

By now, poly (dimethyldiallylammonium chloride) (PDMDAAC), which is the polymer derived from radical homopolymerization of dimethyldiallylammonium chloride (DMDAAC), has been used as the optimum dye fixatives with a view to enhance the uptake of anionic dyes on cotton fabric, and the mechanism of interactions involved can be theoretically interpreted by the participation of electrostatic forces between the dyes and the basic cationic groups in the polymer to reduce the dyes' water-soluble abilities through the formation of color lakes $[3,4]$. However, it appears that no confirmation of the interpretation has been carried out by modern instrumental analysis technologies. Moreover, the cellulose and dimethyldiallylammonium chloride have the similar units of conformational structures in main chains, which would be expected to contribute to close interactions of Van der Waals forces when fixing; thus, PDMDAAC can be widely applied in the fixing of different dyes on cotton fabric [5-8]. However, the development of PDMDAACtreated dyes' rubbing fastness especially wet rubbing fastness is limited; this might be due to the possible dissociation of some of color lakes based on electrostatic forces which are caused by the effect of water molecules and the interactions of Van der Waals forces between PDMDAAC and cotton fabric which are also easy to be destroyed by external 
forces [9]. This promotes researchers to select new series of PDMDAAC-based dye fixatives for further improving the fastness properties of anionic dyes on cotton fabrics.

Some researches indicated that the dye-fixing performances of polycationic dye fixatives could vary from their different molecular weights [10-12], and in our contribution, the authors discovered that those PDMDAAC dye fixatives with the controlled molecular weights characterized by intrinsic viscosities of $0.24-0.47 \mathrm{dL} / \mathrm{g}$ could exhibit better dye-fixing performances [13]. On the other hand, if lower contents (below 20\% molar contents) of reactive units, which can bring about the covalent reactions with the hydroxyl groups of cotton (cellulose) to form a stronger fixing interaction with cotton surface, are incorporated into the backbones of PDMDAAC, the dyefixing performances of those modified PDMDAAC can also be improved [14]. In view of these points in our contribution, a series of novel reactive cationic copolymers of 3-chloro-2-hydroxypropylmethyldiallylammonium chloride (CMDA) and dimethyldiallylammonium chloride (DMDAAC), (P(CMDA-DMDAAC)s) (PDMDAAC-based dye fixatives) with controlled structures of $2 \%-20 \%$ CMDA molar contents in main chains and controlled intrinsic viscosities of $0.15 \sim 0.76 \mathrm{dL} / \mathrm{g}$, which were designed as more useful PDMDAAC-based dye fixatives and derived from the further incorporation of below 20\% molar contents of reactive units (CMDA units) into the backbones of the PDMDAAC with the controlled intrinsic viscosities of $0.24-0.47 \mathrm{dL} / \mathrm{g}$ or nearly, were successfully synthesized (Scheme 1) [15]. However, the information about roles of novel $\mathrm{P}$ (CMDA-DMDAAC)s in fixing anionic dyes on cotton fabric was very limited and especially it has not been verified by modern instrumental analysis in open literature yet. Thus, in this paper, a series of experiments were designed to confirm the roles of novel $\mathrm{P}$ (CMDA-DMDAAC)s in fixing anionic dyes on cotton fabric such as the fixing interactions of $\mathrm{P}(\mathrm{CMDA}-\mathrm{DMDAAC})$ s with anionic dyes and cotton fabric, by modern instrumental analysis technologies [FT-IR spectra and Scanning Electron Microscopy (SEM)], to achieve the new theoretical guides for the wide applications of those PDMDAAC-based dye fixatives.

\section{Experimental}

2.1. Materials and Measurement. All the selected novel $\mathrm{P}(\mathrm{CMDA}-\mathrm{DMDAAC}) \mathrm{s}$ in this paper could be synthesized by the copolymerization of 3-chloro-2-hydroxypropylmethyldiallylammonium chloride (CMDA) and dimethyldiallylammonium chloride (DMDAAC), and by varying molar ratios of raw materials of CMDA to DMDAAC from $2 / 98$ to $20 / 80$ and increasing initial monomer concentrations from $19 \%$ to $40 \%$ with the decrease of initiator amount from $12 \%$ to $6 \%$ during polymerization according to the methods in the literature [15]. Reactive Scarlet 3BS (industrial purity) and cotton fabric (industrial product) were both collected and stored from Jiangsu Nantong Chemicals and Textile Co., Ltd. (China).
The FT-IR spectra of the products in $\mathrm{KBr}$ pellets (2\%) were recorded using a Nicolet FT-IR (510 P) spectrophotometer. Scanning Electron Microscopy (SEM) analysis was measured with a JSM-5610 scanning electron microscopy instrument.

\subsection{Roles of $P(C M D A-D M D A A C)$ s in Fixing Anionic Dyes on Cotton Fabric: Design and Process}

2.2.1. Fixing Interactions of $P(C M D A-D M D A A C) s$ and Anionic Dyes. As polycationic dye fixatives, the basic cationic groups of $\mathrm{P}(\mathrm{CMDA}-\mathrm{DMDAAC}) \mathrm{s}$ were theoretically expected to form the electrostatic forces with anionic dyes and produce the water insoluble color lakes when fixing; however, these explanations have not still been confirmed by modern instrumental analysis. Thus, the color lakes derived from the interactions of one P(CMDA-DMDAAC) containing higher basic cationic DMDAAC units in main chains (the molar contents of DMDAAC units in the main chains were $98 \%$, and the intrinsic viscosity was $0.22 \mathrm{dL} / \mathrm{g}$ ) with one anionic dye (Reactive Scarlet 3BS) were prepared for the studies as one example based on the widely-used basic fixing conditions of PDMDAAC-based dye fixatives on cotton fabric $[13,14]$; the operation processes of forming color lakes were designed and listed as follows.

According to the basic fixing weight ratios of anionic dyes to the PDMDAAC-based dye fixatives $(\mathrm{w} / \mathrm{w}=2 / 3$ ) $[13,14]$, to a $100 \mathrm{~mL}$ baker, $0.50 \mathrm{~g}$ Reactive Scarlet 3BS and $50 \mathrm{~mL}$ deionized water were added, to form a dye solution. And then $0.75 \mathrm{~g}$ of the P(CMDA-DMDAAC) was added with stirring to produce a lot of precipitation (color lake), the precipitation was dried, and the structure was characterized by FT-IR analysis for analyzing the formation of color lakes.

\subsubsection{Fixing Interactions of $P(C M D A-D M D A A C)$ s and Cotton} Fabric. According to the basic fixing conditions of reactive PDMDAAC-based dye fixatives on cotton fabric [14], one of the $\mathrm{P}(\mathrm{CMDA}-\mathrm{DMDAAC}) \mathrm{s}$ containing higher reactive CMDA units in main chains (the molar contents of CMDA units in the main chains were $10 \%$, and the intrinsic viscosity was $0.16 \mathrm{dL} / \mathrm{g}$ ) was selected to be interacted with the undyeing cotton fabric as one example and the structure changes of the untreated and treated cotton samples were confirmed by comparing their FT-IR analysis, for investigating the fixing interactions of $\mathrm{P}$ (CMDA-DMDAAC)s with cotton fabric. Moreover, two higher amounts (50\% o.w.f, and $100 \%$ o.w.f) of the $\mathrm{P}(\mathrm{CMDA}-\mathrm{DMDAAC}) \mathrm{s}$ besides the common fixing amounts (3\% o.w.f) were extraused to treat the undyeing cotton fabric, in order to clearly measure the structure changes of untreated cotton sample and the treated cotton sample by comparing their FT-IR analysis. The operation processes were designed and listed as follows.

Different amounts (3\% o.w.f, $50 \%$ o.w.f, and $100 \%$ o.w.f) of the $\mathrm{P}(\mathrm{CMDA}-\mathrm{DMDAAC}) \mathrm{s}$ were selected to treat the cotton fabric (undyeing) at $60^{\circ} \mathrm{C}$ for $30 \mathrm{~min}$, the $\mathrm{pH}$ of fix application was 7 , the liquor ratio was $1: 20$, and some of the treated cotton samples were directly washed with water for approximately 4-5 times to a constant weight for 

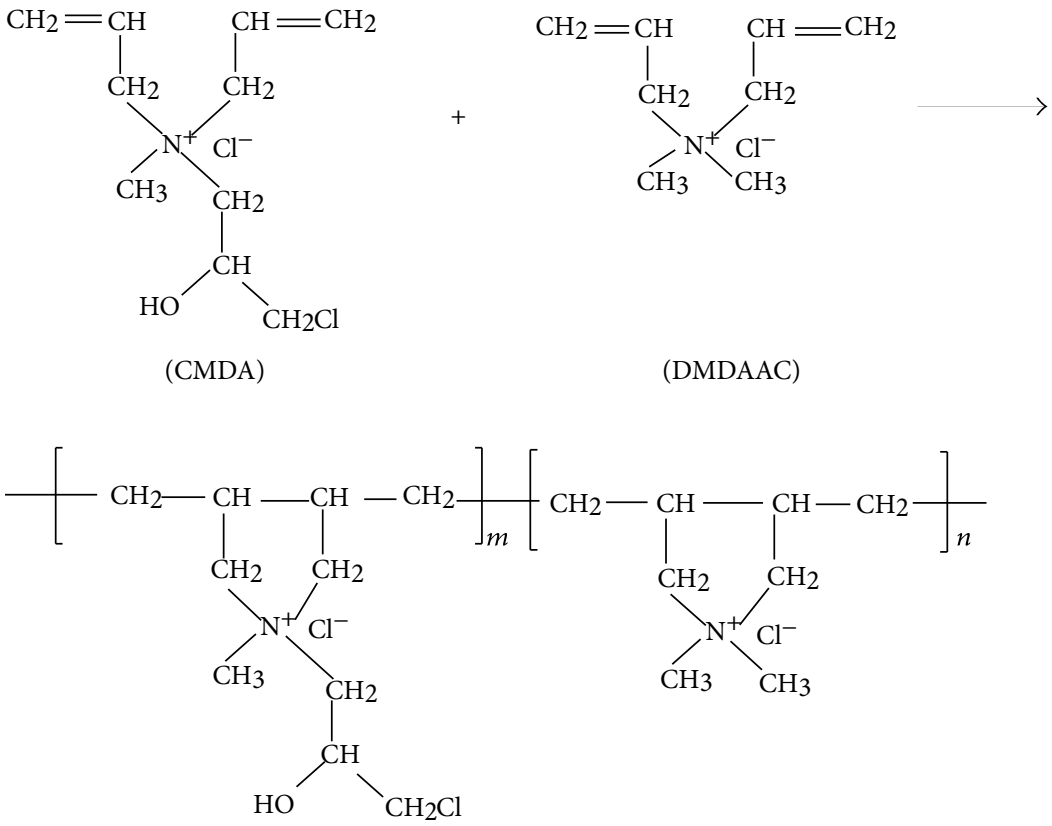

SCHEME 1: Preparation of novel cationic copolymers of $\mathrm{P}(\mathrm{CMDA}-\mathrm{DMDAAC}) \mathrm{s}$ in previous work.

investigating the fixing interaction strengths of the $\mathrm{P}(\mathrm{CMDA}-$ DMDAAC) with cotton fabric and were dried at $50^{\circ} \mathrm{C}$ for $24 \mathrm{~h}$ to be characterized by FT-IR analysis. The treated cotton samples at normal fixing temperature $\left(60^{\circ} \mathrm{C}\right)$ were compared to those of untreated cotton sample, for comparing the structure changes of untreated and treated cotton samples. Then some of another treated cotton samples (unwashed by water) were dried and further baked at $180^{\circ} \mathrm{C}$ for $3 \mathrm{~min}$, washed with water for approximately 4-5 times to a constant weight for their fixing investigating interaction strengths of the P(CMDA-DMDAAC) with cotton fabric, and dried to be characterized by FT-IR analysis. This sample, as the treated cotton sample baked at the higher temperature, was compared to that of untreated cotton sample, for comparing the structure changes of untreated and treated cotton samples at the normal fixing temperature.

\subsubsection{Fixing Interactions of $P(C M D A-D M D A A C)$ s on Dyeing Cotton Fabric}

2.2.3.1. Fixing Interactions of $P(C M D A-D M D A A C) s$ and Anionic Dyes on Dyeing Cotton Fabric. According to the basic dyeing and fixing conditions of reactive PDMDAACbased dye fixatives on cotton fabric (Scheme 2) [14], one of the $\mathrm{P}(\mathrm{CMDA}-\mathrm{DMDAAC}) \mathrm{s}$ containing higher basic cationic DMDAAC units in main chains (the molar contents of DMDAAC units in the main chains were $98 \%$, and the intrinsic viscosity was $0.22 \mathrm{dL} / \mathrm{g}$ ) was selected to fix one anionic dye (Reactive Scarlet 3BS) on dyeing cotton fabric as one example. And the structure changes of the selected anionic dyes on unfixed and fixed dyeing cotton samples were confirmed by comparing their FT-IR analysis, for investigating the fixing interactions of $\mathrm{P}(\mathrm{CMDA}-\mathrm{DMDAAC})$ s with anionic dyes on dyeing cotton fabric. Moreover, a higher amount (100\% o.w.f) of Reactive Scarlet 3BS is expected for the common dyeing amounts (2\% o.w.f) and higher amounts (150\% o.w.f, and $300 \%$ o.w.f) of the P(CMDA-DMDAAC)s besides the common fixing amounts (3\% o.w.f) were selected, in order to clearly measure the structure changes of dyes on dyeing cotton fabric by comparing their FT-IR analysis. The operation processes were designed and listed as follows.

As shown in Scheme 2, the cotton fabric was dyed with $100 \%$ (o.w.f) Reactive Scarlet $3 \mathrm{BS}$ at $60^{\circ} \mathrm{C}$, and then different amounts (3\% o.w.f, $150 \%$ o.w.f, and $300 \%$ o.w.f) of the $\mathrm{P}(\mathrm{CMDA}-\mathrm{DMDAAC}) \mathrm{s}$ were respectively selected to fix the selected anionic dye on the dyeing cotton fabric at $60^{\circ} \mathrm{C}$ for $30 \mathrm{~min}$, the $\mathrm{pH}$ of fix application was 7 , the liquor ratio was $1: 20$, and then the fixed dyeing cotton samples were dried to be characterized by FT-IR analysis for comparing the structure changes of the unfixed dyeing cotton fabric and dyeing cotton fabric fixed by the $\mathrm{P}(\mathrm{CMDA}-\mathrm{DMDAAC})$.

\subsubsection{Fixing Interactions of $P(C M D A-D M D A A C) s$ and} Dyeing Cotton Fabric. According to the basic dyeing and fixing conditions of reactive PDMDAAC-based dye fixatives on cotton fabric [14], one of the P(CMDA-DMDAAC) containing higher reactive CMDA units in main chains (the molar contents of CMDA units in the main chains were $10 \%$, and the intrinsic viscosity was $0.16 \mathrm{dL} / \mathrm{g}$ ) was used to fix the dyeing cotton fabric as one example and the surface morphologies of unfixed dyeing cotton sample and the $\mathrm{P}(\mathrm{CMDA}-\mathrm{DMDAAC})$-fixed dyeing cotton samples were confirmed by comparing their Scanning Electron Microscopy (SEM) analysis, for further investigating the fixing interactions of $\mathrm{P}(\mathrm{CMDA}-\mathrm{DMDAAC})$ s with dyeing cotton fabric. The operation processes were designed and listed as follows. 


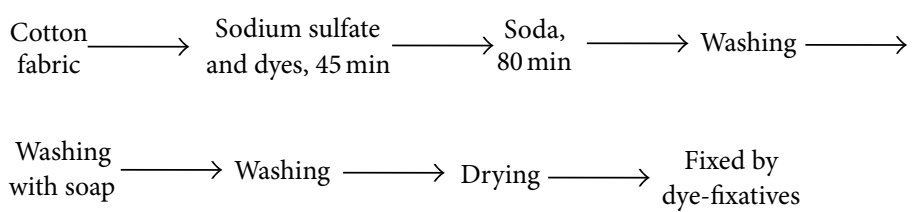

Scheme 2: Dyeing and fixing processes on cotton fabric.

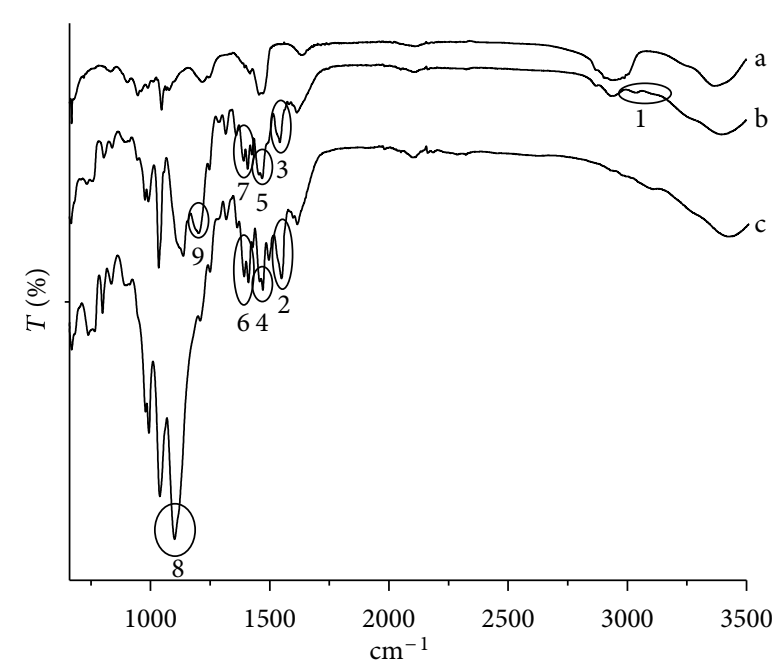

FIGURE 1: FT-IR analysis of color lakes derived from the interaction between one P(CMDA-DMDAAC) and dyes. (a) P(CMDADMDAAC); (b) the color lakes; (c) Reactive Scarlet 3BS.

As shown in Scheme 2, the cotton fabric was dyed with $2 \%$ (o.w.f) Reactive Scarlet 3BS at $60^{\circ} \mathrm{C}$, and then 3\% (o.w.f) of the $\mathrm{P}(\mathrm{CMDA}-\mathrm{DMDAAC}) \mathrm{s}$ were used to treat the cotton fabric dyed with Reactive Scarlet $4 \mathrm{BS}$ at $60^{\circ} \mathrm{C}$ for $30 \mathrm{~min}$; the $\mathrm{pH}$ of fix application was 7 ; the liquor ratio was $1: 20$; moreover, the fixed dyeing cotton sample was dried at room temperature and then further baked at $180^{\circ} \mathrm{C}$ for $3 \mathrm{~min}$ [14]. Finally, Scanning Electron Microscopy (SEM) analysis was used to characterize the surface morphologies of unfixed dyeing cotton sample and the P(CMDA-DMDAAC)-fixed dyeing cotton sample [16].

\section{Results and Discussion}

3.1. Interactions of $P(C M D A-D M D A A C)$ s and Anionic Dyes. As mentioned in Section 2.2.1, the FT-IR analysis of color lakes derived from the interaction between one P(CMDADMDAAC) (the molar contents of CMDA units in the main chains were $2 \%$, and the intrinsic viscosity was $0.22 \mathrm{dL} / \mathrm{g}$ ) and Reactive Scarlet 3BS was shown in Figure 1.

Figure 1 showed the FT-IR spectra of the color lakes had the absorptions at approximately $728 \mathrm{~cm}^{-1}-1032 \mathrm{~cm}^{-1}$ which were similar to those of Reactive Scarlet 3BS, indicating that their structures consisted of dyes (Reactive Scarlet 3BS), and had the absorptions of the $-\mathrm{CH}_{3}$ groups at $3039 \mathrm{~cm}^{-1}$, the absorptions of the $-\mathrm{CH}_{2}-$ linkage at $2936 \mathrm{~cm}^{-1}$, and the absorptions of the methyne linkage at $2863 \mathrm{~cm}^{-1}$, which

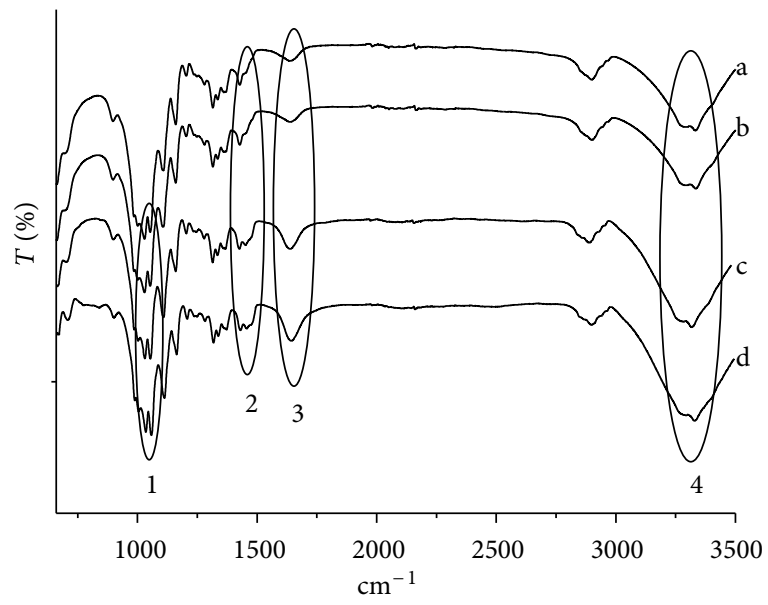

FIGURE 2: FT-IR analysis of untreated and treated cotton (unwashed). (a) Unfixed cotton sample; (b) the cotton sample fixed by $3 \%$ (o.w.f) slightly cross-linked PDMDAAC; (c) the cotton sample fixed by $50 \%$ (o.w.f) slightly crosslinked PDMDAAC; (d) the cotton sample fixed by $100 \%$ (o.w.f) slightly crosslinked PDMDAAC.

were similar to those of $\mathrm{P}(\mathrm{CMDA}-\mathrm{DMDAAC})$, indicating that their structures consisted of the P(CMDA-DMDAAC) (Peak 1).

In addition, after forming color lakes, the dye's absorptions at $1549 \mathrm{~cm}^{-1}$ (Peak 2), $1562 \mathrm{~cm}^{-1}$ (Peak 4), $1407 \mathrm{~cm}^{-1}$, and $1390 \mathrm{~cm}^{-1}$ (Peak 6) were, respectively, shifted to $1541 \mathrm{~cm}^{-1}$ (Peak 3), $1568 \mathrm{~cm}^{-1}$ (Peak 5), $1417 \mathrm{~cm}^{-1}$, and $1394 \mathrm{~cm}^{-1}$ (Peak 7); especially the absorption of dye's sulfonate anion at $1103 \mathrm{~cm}^{-1}$ (Peak 8) was shifted to $1202 \mathrm{~cm}^{-1}$ (Peak 9); those dye's structure changes could be attributed to the electrostatic interactions between the P(DHACDMDAAC) and the anionic dye (Reactive Scarlet 3BS).

Thus, it could be concluded from the above-mentioned results that the basic cationic groups of the obtained $\mathrm{P}$ (CMDA-DMDAAC)s would form electrostatic forces with anionic dyes to produce the insoluble-water color lakes.

\subsection{Interactions of $P(C M D A-D M D A A C)$ s and Cotton Fabric}

3.2.1. Interactions of $P(C M D A-D M D A A C)$ s and Cotton Fabric at Normal Fixing Temperatures. As mentioned in Section 2.2.2, the FT-IR analysis of untreated cotton samples and the cotton samples treated by the P(CMDA-DMDAAC)s at normal fixing temperatures was shown in Figures 2 and 3.

Figure 2 showed the FT-IR spectra of the untreated cotton fabric had the absorption of the $\mathrm{OH}$ group at $3349 \mathrm{~cm}^{-1}$, the 


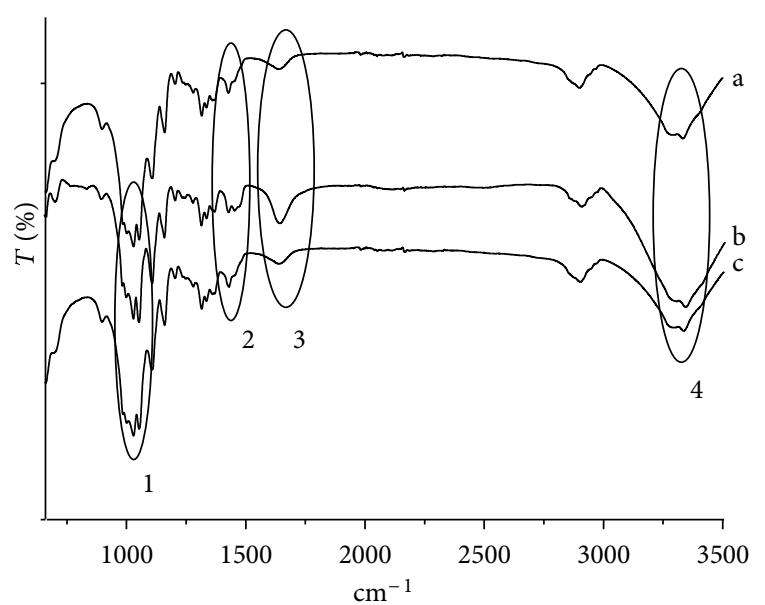

FIGURE 3: FT-IR analysis of untreated and treated cotton (washed). (a) Unfixed cotton sample; (b) the cotton sample fixed by $100 \%$ (o.w.f) slightly crosslinked PDMDAAC (unwashed); (c) the cotton sample fixed by $100 \%$ (o.w.f) slightly crosslinked PDMDAAC (washed).

absorption of the $-\mathrm{CH}$ linkage at $2901 \mathrm{~cm}^{-1}$, the absorption of the $-\mathrm{CH}_{2}-$ group at $1435 \mathrm{~cm}^{-1}$, and the absorption of the C-O-C group at $1029 \mathrm{~cm}^{-1}, 1053 \mathrm{~cm}^{-1}, 1108 \mathrm{~cm}^{-1}$, and $1160 \mathrm{~cm}^{-1}$, indicating that cotton fabric was mainly made of cellulose [16].

As well as the similar absorptions to the untreated cotton fabric, the FT-IR spectra of the cotton samples fixed by the $\mathrm{P}(\mathrm{CMDA}-\mathrm{DMDAAC})$ further showed the new absorptions at $1428 \mathrm{~cm}^{-1}$ of the P(CMDA-DMDAAC) (Peak 2); moreover, the absorptions at $1053 \mathrm{~cm}^{-1}$ (Peak 1), $1640 \mathrm{~cm}^{-1}$ (Peak 3 ), and $3349 \mathrm{~cm}^{-1}$ (Peak 4) were all increased with the increase of the amount of $\mathrm{P}$ (DHAC-DMDAAC), because the $\mathrm{P}(\mathrm{CMDA}-\mathrm{DMDAAC})$ had also the similar absorptions at those shifts, which would increase the intensity of the similar absorptions. Thus, all these results suggested that the $\mathrm{P}(\mathrm{CMDA}-\mathrm{DMDAAC}) \mathrm{s}$ could be penetrated into cotton fabric and be convenient to interact with anionic dyes when fixing.

However, after being adequately washed with water to a constant weight (Figure 3), the new absorptions of the $\mathrm{P}$ (DHAC-DMDAAC) at $1428 \mathrm{~cm}^{-1}$ would be absent, and the increased absorptions $\left(1053 \mathrm{~cm}^{-1}, 1640 \mathrm{~cm}^{-1}\right.$, and $3349 \mathrm{~cm}^{-1}$ ) were all decreased so that they were similar to those of untreated cotton fabric, possibly indicating that most of the interactions of the P(DHAC-DMDAAC) and cotton fabric were attributed to Van der Waals forces at lower fixing temperatures (only $60^{\circ} \mathrm{C}$ ), which were very weak [5].

3.2.2. Interactions of $P(C M D A-D M D A A C)$ s and Cotton Fabric Baked at Higher Temperatures. As mentioned in Section 2.2.2, the FT-IR analysis of untreated cotton sample and the cotton sample treated by $\mathrm{P}(\mathrm{CMDA}-\mathrm{DMDAAC})$ s baked at $180^{\circ} \mathrm{C}$ was shown in Figure 4 .

Figure 4 showed as well as the similar absorptions to the untreated cotton fabric, compared to the results at normal fixing temperatures (Figure 3), even if being washed with

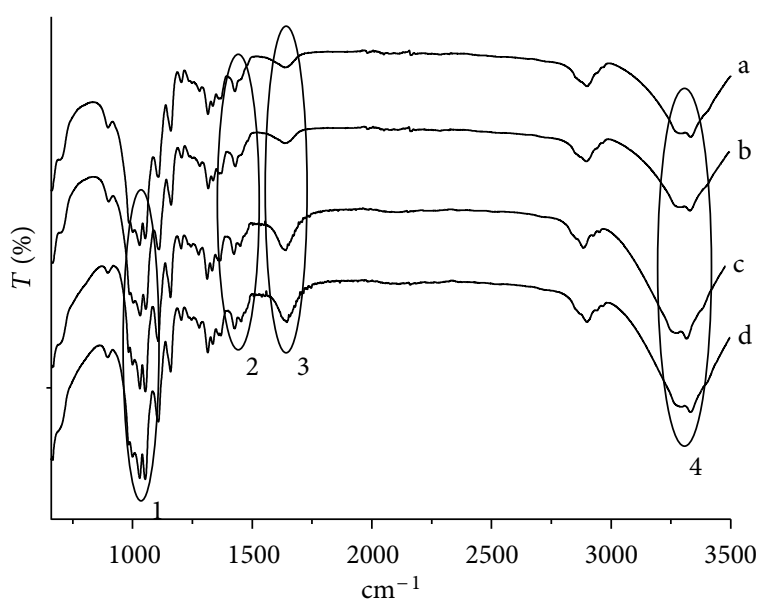

FIGURE 4: FT-IR analysis of untreated and treated cotton (washed). (a) Untreated cotton sample (undyeing); (b) the cotton sample (undyeing) treated by 3\% (o.w.f) P(CMDA-DMDAAC); (c) the cotton sample (undyeing) treated by $50 \%$ (o.w.f) P(CMDADMDAAC); (d) the cotton sample (undyeing) treated by $100 \%$ (o.w.f) P(CMDA-DMDAAC).

water for approximately 4-5 times to a constant weight, the FT-IR spectra (Curve "c" and Curve "d") of the cotton samples fixed by the $\mathrm{P}(\mathrm{CMDA}-\mathrm{DMDAAC})$ further showed that the absorption intensity of the P(DHAC-DMDAAC)treated cotton sample at $1053 \mathrm{~cm}^{-1}$ (Peak 1) was higher than that of untreated cotton fabric (Curve "a"); this might be due to the formation of new $\mathrm{C}-\mathrm{O}-\mathrm{C}$ linkage, derived from the reaction linkage between the reactive units (CMDA) of the obtained $\mathrm{P}(\mathrm{CMDA}-\mathrm{DMDAAC}) \mathrm{s}$ and the hydroxyl groups of cotton (cellulose), resulting in the increase of the intensity of the similar absorptions. In addition, the absorptions at $1429 \mathrm{~cm}^{-1}$ (Peak 2), $1640 \mathrm{~cm}^{-1}$ (Peak 3), and $3349 \mathrm{~cm}^{-1}$ (Peak 4) were still increased with the increase of the amount of $\mathrm{P}(\mathrm{CMDA}-\mathrm{DMDAAC})$ as similar as shown in Figure 2; further indicating that a strong interaction could link the $\mathrm{P}(\mathrm{CMDA}-$ DMDAAC) and the cotton fabric.

Thus, it could be concluded from the above-mentioned results that the reactive units (CMDA) of the obtained $\mathrm{P}(\mathrm{CMDA}-\mathrm{DMDAAC}) \mathrm{s}$ could be possibly expected to bring about covalent bonds with the hydroxyl groups of cotton (cellulose) to form a new ether linkage, resulting in the stronger interactions of $\mathrm{P}(\mathrm{CMDA}-\mathrm{DMDAAC})$ s with cotton fabric when fixing.

\subsection{Interactions of $P(C M D A-D M D A A C)$ s on Dyeing Cotton Fabric}

3.3.1. Interactions of $P(C M D A-D M D A A C)$ s and Anionic Dyes on Dyeing Cotton Fabric. As mentioned in Section 2.2.3.1, the FT-IR analysis of unfixed dyeing cotton sample and the cotton sample fixed by different amounts (3\% o.w.f, $150 \%$ o.w.f, and $300 \%$ o.w.f) of P(CMDA-DMDAAC)s was shown in Figure 5. 


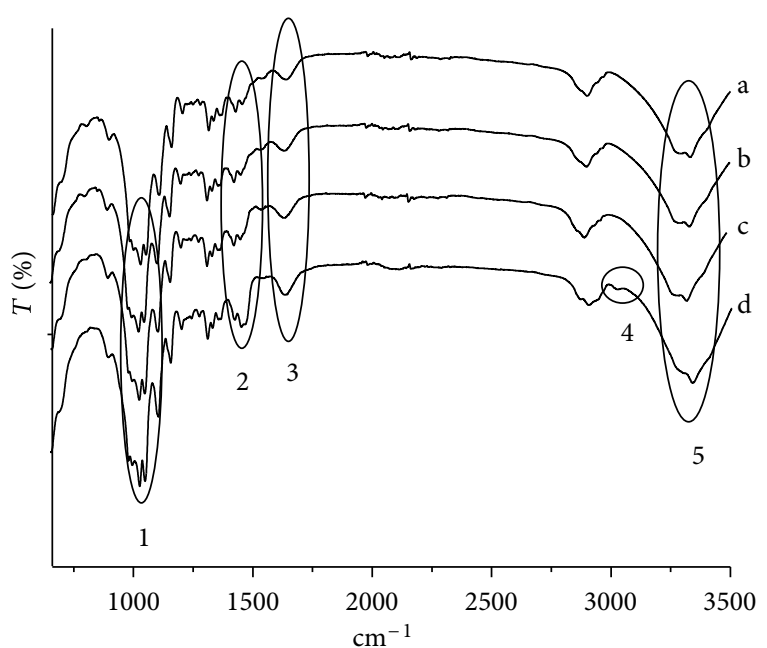

FIGURE 5: FT-IR analysis of unfixed and fixed dyeing cotton (unwashed). (a) Unfixed dyeing cotton sample; (b) the dyeing cotton sample fixed by $3 \%$ (o.w.f) P(CMDA-DMDAAC); (c) the dyeing cotton sample fixed by $150 \%$ (o.w.f) P(CMDA-DMDAAC); (d) the dyeing cotton sample fixed by $300 \%$ (o.w.f) P(CMDA-DMDAAC).

Figure 5 showed, the FT-IR spectra of dyeing cotton samples had the dye's absorptions at $802 \mathrm{~cm}^{-1}, 1456 \mathrm{~cm}^{-1}$, and $1543 \mathrm{~cm}^{-1}$, and the absorption of dye's sulfonate anion at $1103 \mathrm{~cm}^{-1}$ or nearly was blocked by the similar strong absorption of C-O-C linkage of cotton (cellulose).

Compared to those of unfixed dyeing cotton sample, the FT-IR spectra of the dyeing cotton sample fixed by $300 \%$ (o.w.f) P(CMDA-DMDAAC) had the absorption of the $-\mathrm{CH}_{3}$ groups at $3022 \mathrm{~cm}^{-1}$ (Peak 1, Curve "d"), which could be attributed to the $\mathrm{P}$ (CMDA-DMDAAC). Moreover, the absorptions at $1456 \mathrm{~cm}^{-1}$ (Peak 3) and $1053 \mathrm{~cm}^{-1}$ (Peak 4) were both increased with the increase of the amount of $\mathrm{P}$ (CMDA-DMDAAC), because the P(CMDA-DMDAAC) had also the similar absorptions at the corresponding shifts, which would increase the intensity of the similar absorptions. Therefore, all these results further indicated that P(CMDADMDAAC)s could be penetrated into cotton fabric and be convenient to interact with dyes when fixing.

In addition, similar to the results of forming color lakes (Section 3.1), the FT-IR spectra of dyeing cotton samples fixed by the $\mathrm{P}(\mathrm{CMDA}-\mathrm{DMDAAC})$ s further also showed that the absorption of the dye on dyeing cotton sample was shifted from $1543 \mathrm{~cm}^{-1}$ to $1540 \mathrm{~cm}^{-1}$ (Peak 2, Curve "d" in Figure 5), which was in agreement with that of forming color lakes (Section 3.1), further indicating that the expected color lakes could be possibly formed on dyeing cotton fabric, to play a role in the development of fastness of dyes on cotton fabric.

3.3.2. Interactions of $P(C M D A-D M D A A C)$ s and Dyeing Cotton Fabric. As mentioned in Section 2.2.3.2, Scanning Electron Microscopy (SEM) analysis was used to characterize the surface morphologies of unfixed dyeing cotton sample and the dyeing cotton sample fixed by the P(CMDA-DMDAAC) (the molar contents of CMDA units in main chains were $10 \%$,

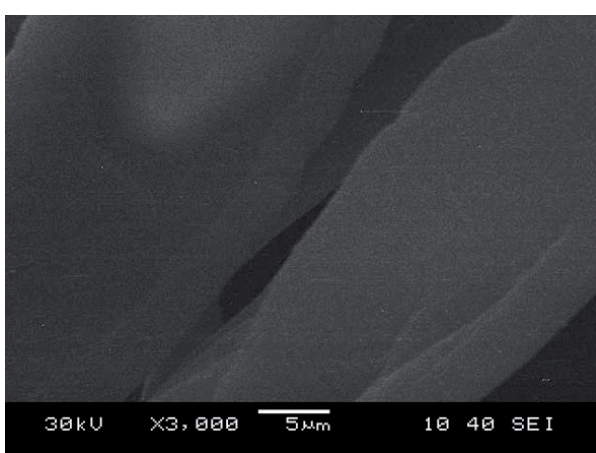

(a)

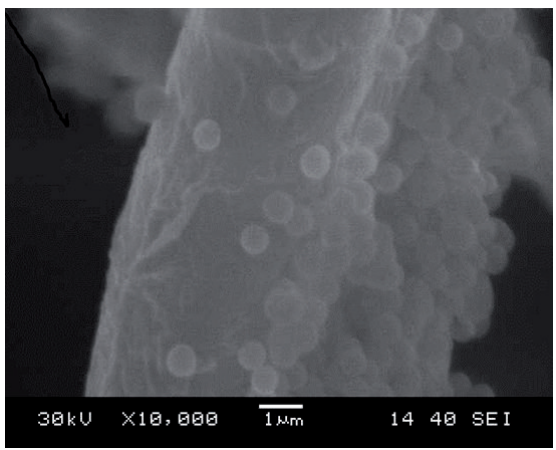

(b)

Figure 6: SEM analysis of unfixed and fixed dyeing cotton. (a) Untreated dyeing cotton sample; (b) $\mathrm{P}(\mathrm{CMDA}-\mathrm{DMDAAC})$-fixed dyeing cotton sample.

and the intrinsic viscosity was $0.16 \mathrm{dL} / \mathrm{g}$ ); the results were shown in Figure 6.

Figure 6 showed, compared to the result of unfixed dyeing cotton sample, there were a lot of new surface materials possibly derived from the fixing participation of $\mathrm{P}(\mathrm{CMDA}$ DMDAAC), which closely adhered to the surface of the fixed dyeing cotton sample, indicating that they could be convenient to form the further fixing interactions of $\mathrm{P}(\mathrm{CMDA}$ DMDAAC)s with cotton fabric such as covalent bonds.

\section{Conclusions}

It could be verified by the studies on the roles of novel reactive cationic copolymers (P(CMDA-DMDAAC)s) of 3-chloro-2-hydroxypropylmethyldiallylammonium chloride and dimethyldiallylammonium chloride in fixing anionic dyes on cotton fabric, using several modern instrumental analysis technologies (FT-IR spectra and SEM analysis), that the reactive units (CMDA) of the obtained $\mathrm{P}$ (CMDADMDAAC)s could be expected to bring about the covalent bonds with the hydroxyl groups of cotton (cellulose) to form an ether linkage at higher temperature $\left(180^{\circ} \mathrm{C}\right)$, resulting in the stronger fixing interactions of $\mathrm{P}(\mathrm{CMDA}-\mathrm{DMDAAC}) \mathrm{s}$ with cotton fabric, besides their basic cationic groups would form electrostatic forces with anionic dyes to produce the insoluble-water color lakes, for the development of dyes' fastness on cotton fabric. 


\section{References}

[1] H. F. Wang, Y. Y. Chen, and H. Lin, "The research history and development trend of cotton fiber," Journal of Soochow University Engineering Science Edition, vol. 23, no. 4, pp. 12-19, 2003.

[2] J. Zhang, "Situation and development trends of dyestuffs used for cellulose fibres in the world," Fine and Specialty Chemicals, vol. 12, no. 8, pp. 1-5, 2004.

[3] C. Wandrey, B. J. Hernandez, and D. Hunkerler, "Diallyildimethylammonium chloride and its polymers," Advances in Polymer Science, vol. 145, pp. 123-182, 1999.

[4] S. Rosunee, C. M. Carr, S. Hibbert, and C. Jones, "Surface chemical analysis of Tencel treated with a cationic fixing agent," Journal of Materials Science, vol. 38, no. 10, pp. 2179-2185, 2003.

[5] S. M. Burkinshaw and A. Gotsopoulos, "Pretreatment of cotton to enhance its dyeability; part 2. Direct dyes," Dyes and Pigments, vol. 42, no. 2, pp. 179-195, 1999.

[6] S. M. Burkinshaw and A. Gotsopoulos, "The pre-treatment of cotton to enhance its dyeability-I. Sulphur dyes," Dyes and Pigments, vol. 32, no. 4, pp. 209-228, 1996.

[7] K. Shinbo, K. Onishi, S. Miyabayashi et al., "Fabrication and electrochemical properties of layer-by-layer deposited films containing phthalocyanine dyes," Thin Solid Films, vol. 438-439, pp. 177-181, 2003.

[8] G. Nizri, S. Lagerge, A. Kamyshny, D. T. Major, and S. Magdassi, "Polymer-surfactant interactions: binding mechanism of sodium dodecyl sulfate to poly(diallyldimethylammonium chloride)," Journal of Colloid and Interface Science, vol. 320, no. 1, pp. 74-81, 2008.

[9] S. M. Burkinshaw and D. Katsarelias, "A study of the wash-off and aftertreatment of dichlorotriazinyl reactive dyes on cotton," Dyes and Pigments, vol. 29, no. 2, pp. 139-153, 1995.

[10] W. X. Li, H. X. Hong, and H. Zhang, "Preparation and application of reactive non-formaldehyde fixing agent on cotton fabric," Journal of Beijing Institute of Clothing Technology, vol. 28, no. 2, pp. 19-24, 2008.

[11] K. Komiya, S. Kanai, and K. Beppu, "Agents for improving fastness of reactive-dyed fabrics," JP 63264 985, 1988-11-1.

[12] J. G. Zhang, "Study on simplifying the preparing process of cationic polyelectrolytic dye fixative," Fine Petrochemical Industry, no. 1, pp. 36-38, 1992.

[13] Y. K. Yu and Y. J. Zhang, "Molecular-weight-controlled synthesis and dye-fixing properties of poly(dimethyldiallylammonium chloride)," Journal of Vinyl and Additive Technology, vol. 16, no. 4, pp. 277-283, 2010.

[14] H. T. Yang, X. D. Zhou, J. Dong et al., "Synthesis and application of fixing agent AM-DMDAAC copolymer," Dye Printing, no. 5, pp. 10-13, 2010.

[15] Y. Yu and Y. Zhang, "Controlled-synthesis of novel reactive cationic copolymers of 3-chloro-2-hydroxypropylmethyldiallylammonium chloride and dimethyldiallylammonium chloride $[\mathrm{P}(\mathrm{CMDA}-\mathrm{DMDAAC}) \mathrm{s}]$ : designed as useful polycationic dye-fixatives on cotton fabric," Researches on Chemical Intermediates, vol. 38, no. 8, pp. 2097-2109, 2012.

[16] S. L. Cui, Synthesis and Application of Crosslinker TETS, Donghua University, Shanghai, China, 2005. 

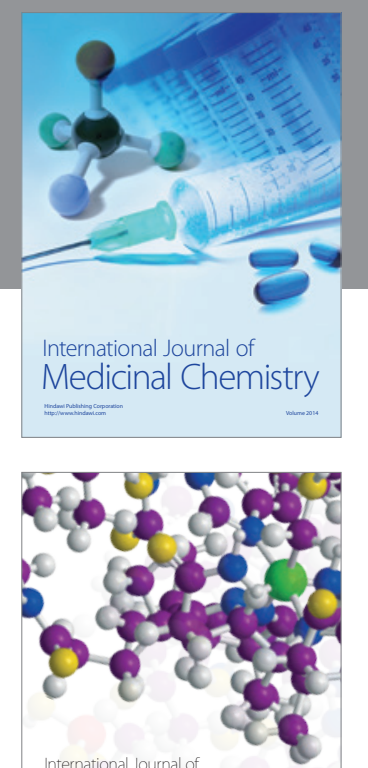

\section{Carbohydrate} Chemistry

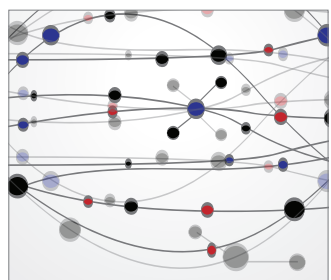

The Scientific World Journal
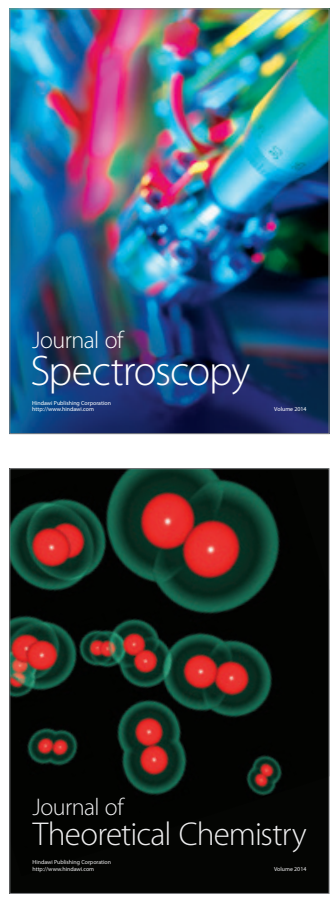
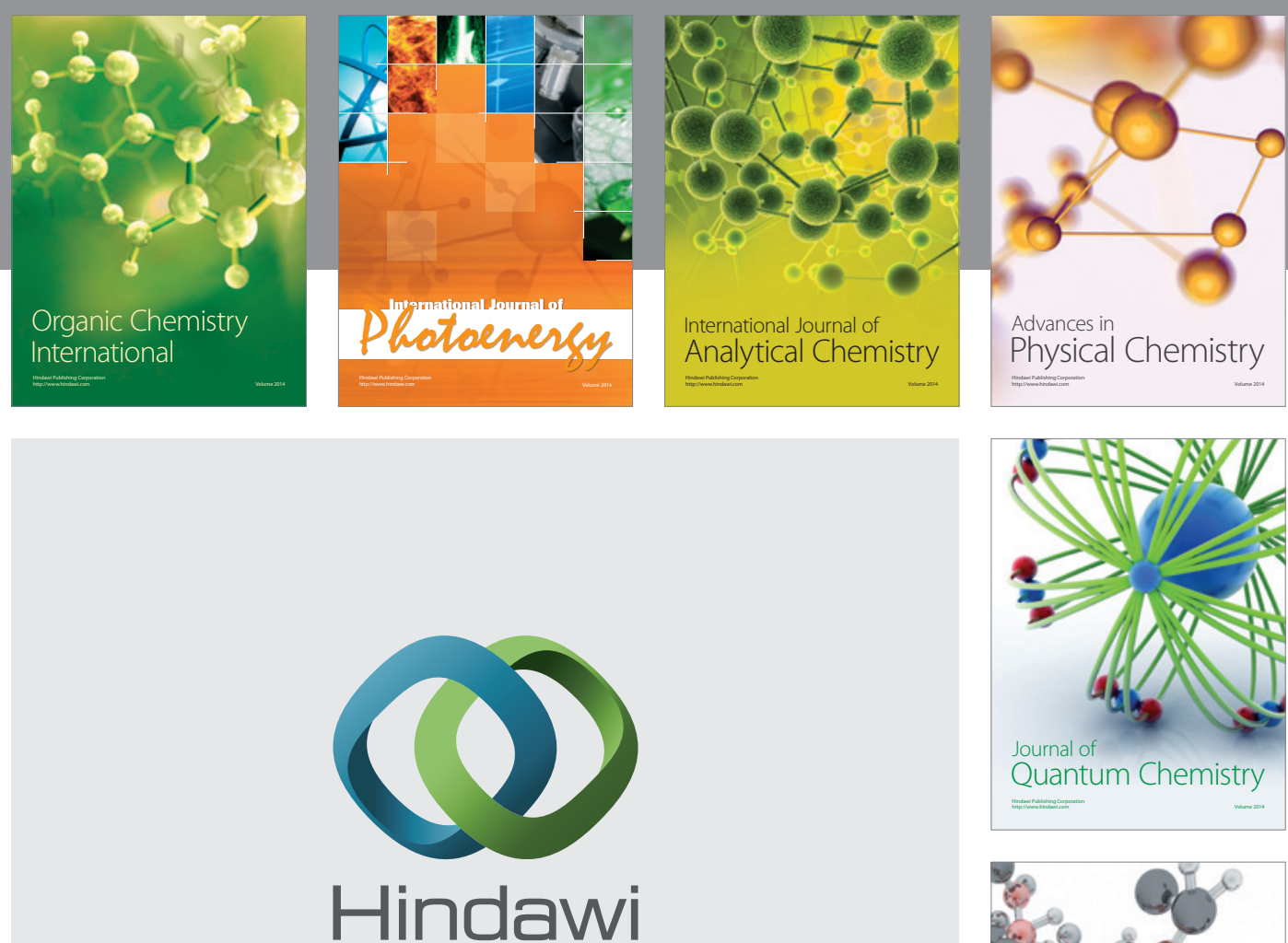

Submit your manuscripts at

http://www.hindawi.com

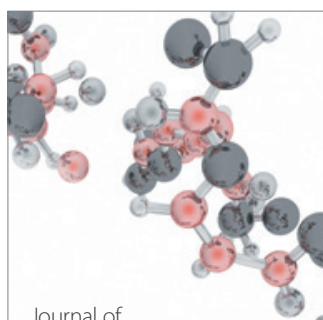

Analytical Methods

in Chemistry

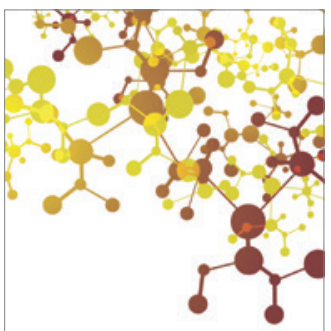

Journal of

Applied Chemistry

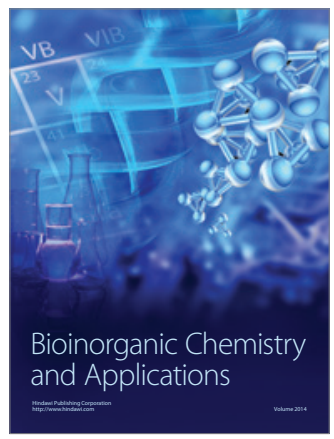

Inorganic Chemistry
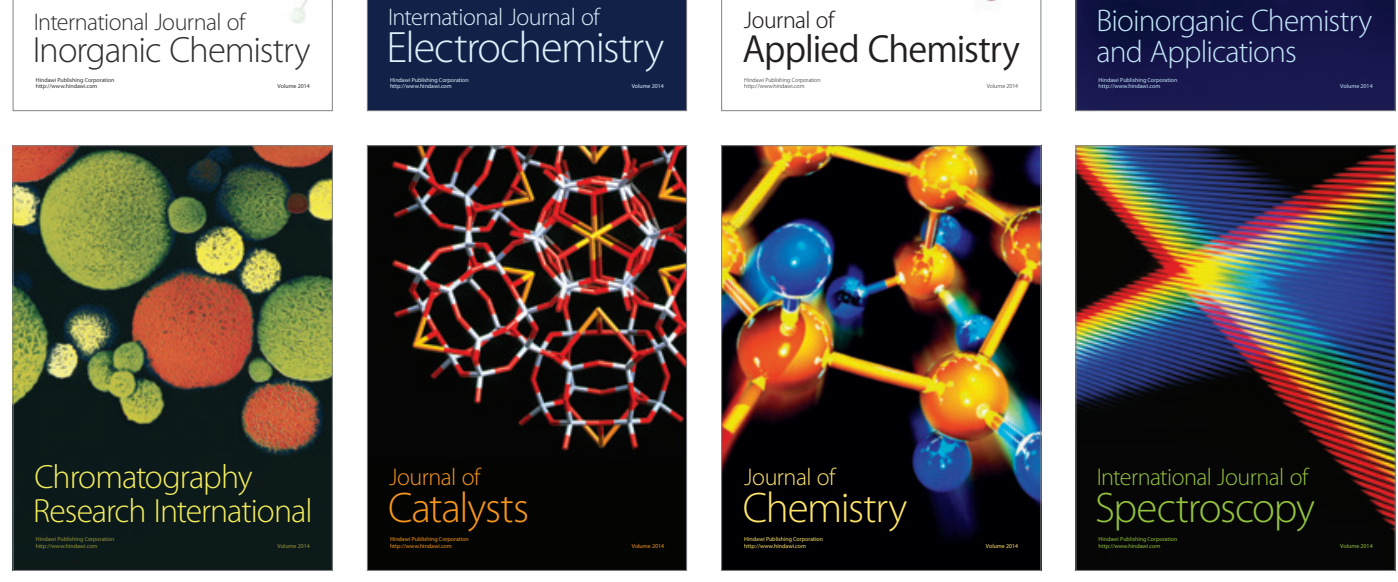